\title{
Features for micro-Doppler based activity classification
}

Svante Björklund, Henrik Petersson and Gustaf Hendeby

\section{Linköping University Post Print}

\section{Tweet}

N.B.: When citing this work, cite the original article.

Original Publication:

Svante Björklund, Henrik Petersson and Gustaf Hendeby, Features for micro-Doppler based activity classification, 2015, IET radar, sonar \& navigation, (9), 9, 1181-1187.

http://dx.doi.org/10.1049/iet-rsn.2015.0084

Copyright: Institution of Engineering and Technology

http://www.theiet.org/

Postprint available at: Linköping University Electronic Press

http://urn.kb.se/resolve?urn=urn:nbn:se:liu:diva-123186 


\title{
On Features for
}

\section{Micro-Doppler Based Activity Classification}

\author{
Henrik Petersson $^{1}$, Svante Björklund ${ }^{1,3}$ and Gustaf Hendeby ${ }^{1,2}$ \\ ${ }^{1}$ Swedish Defence Research Institute (FOI), SE-581 11, Linköping, Sweden. email: \\ henrik.petersson@foi.se, svante.bjoerklundefoi.se \\ ${ }^{2}$ Linköping University, SE-581 83 Linköping, Sweden. email: hendeby@isy.liu.se \\ ${ }^{3}$ Blekinge Institute of Technology, SE-371 79 Karlskrona, Sweden.
}

\begin{abstract}
Safety and security applications benefit from better situational awareness. Radar micro-Doppler signatures from an observed target carry information about the target's activity, and have potential to improve situational awareness. This article describes, compares, and discusses two methods to classify human activity based on radar micro-Doppler data. The first method extracts physically interpretable features from the time-velocity domain such as the main cycle time and properties of the envelope of the micro-Doppler spectra and use these in the classification. The second method derives its features based on the components with the most energy in the cadence-velocity domain (obtained as the Fourier transform of the time-velocity domain). Measurements from a field trial show that the two methods have similar activity classification performance. It is suggested that target base velocity and main limb cadence frequency are indirect features of both methods, and that they do often alone suffice to discriminate between the studied activities. This is corroborated by experiments with a reduced feature set. This opens up for designing new more compact feature sets. Also, weaknesses of the methods and the impact of non-radial motion are discussed.
\end{abstract}

\section{Keywords}

Human activity classification, micro-Doppler radar, micro-Doppler feature extraction, remote monitoring.

\section{INTRODUCTION}

The world today has brought on a need to pay increased attention to safety and security issues, e.g., search and rescue operations, surveillance, and protection of critical infrastructure. These tasks are often labor intensive and potentially dangerous. This provides an incentive to create systems that aid operators to gain situational awareness.

Part of gaining situational awareness is to know what the people in an area are doing. This article addresses this problem by studying how to classify human activity using radar micro-Doppler measurements. The microDoppler measurements provide valuable information about the movements of different body parts, primarily the legs, arms, and torso. Compared to electro-optical (EO) sensors, radar offers better range and radial velocity information, as well as, insensitivity to lighting and weather conditions, at the cost of lower cross-range resolution. This motivates using a radar system.

The classification problem is often divided into two subproblems: feature extraction and classification. The latter is usually performed using standard methods. Feature extraction is very problem specific, and depends on factors such as the type of the radar used, the environment, and target characteristics. A wide variety of radar 
systems have been applied to the problem [1-6], contributing to a variety of different suggested features. Two main types of features are mostly pursued in literature: features derived directly from the received radar signal [7-10] and features computed from time-velocity-diagrams (TVDs) $[1-3,6,11,12]$. The former features are often inspired by similar approaches used in speech processing.

In [9] human activities of a human standing still were classified. Fighting and/or if a target is carrying an object has also been studied in the literature [1,3,11]; as well as, human activities including groups of people or vehicles [2]. The focus here is to study methods that distinguish between a number of different human activities; creeping, crawling, walking, jogging, and running, similar to [1].

TVDs can be used in several ways for classification: direct comparison to average class TVDs [11]; extraction of features using principal component analysis (PCA) and linear discriminant analysis (LDA) [3, 11]; or extraction of features with physical interpretation $[1,6]$. Another approach is to derive cadence velocity diagrams (CVDs) from the TVDs and extract features directly from the CVD [4,5]; or further process the CVDs to remove effects of the base velocity [2].

Several different classifiers have been used for micro-Doppler classification, e.g., support vector machines (SVMs) [1, 2, 4, 6, 13], Gaussian mixture models (GMMs) [2], Bayesian classifier [14] and k-nearest neighbour classifier [13,14]. Overall, a classification accuracy of approximately $90 \%$ is reported.

Here, a TVD based method with interpretable features [1] and a CVD based method [4, 5] will be described, compared, and discussed based on micro-Doppler datasets of people performing different activities. Based on this, two major information carriers in the dataset are pointed out, the target base velocity and the main limb cadence frequency, which together are enough to achieve classification performance comparable with the two other studied, and considerably more complex, methods. The two information carriers are present in both the more complex methods. In this way we hope to highlight the question: How much information must a feature set contain to be useful. Neither of the two methods is new. Instead the novelty of this article is the comparison of the two methods; and based on the fact that they perform similarly, a discussion about what underlying properties are the most important for the classification.

The article is organized in the following way. In Sec. II the addressed problem and the available datasets are described, Sec. III deals with the classification problem with focus on the feature extraction step and the two studied methods. Sec. IV describes how the experimental results are obtained. The results are then presented in Sec. V and discussed in Sec. VI and the findings are summarized in Sec. VII.

\section{Available Data}

\section{A. Radar Description}

The radar system used is a frequency modulated continuous wave (FMCW) radar with linear FM sweeps (40 $\mu \mathrm{s}$ length and $160 \mathrm{MHz}$ bandwidth), a PRF (the repetition frequency of the sweeps) of $17.6 \mathrm{kHz}$, a carrier frequency of $77 \mathrm{GHz}$ and horizontal polarization (called SIRS1600TD) by SAAB AB. Its designed target detection range is between $10 \mathrm{~m}$ and $200 \mathrm{~m}$. The radar has a resolution of $1 \mathrm{~m}$ in range and $1^{\circ}$ in azimuth. In our setup, the radar was pointing fairly horizontal. The sensor is a prototype of a general purpose mobile radar system. It is neither specifically optimized for obtaining micro-Doppler, nor for the specific classification task. The fact that the radar was not designed specifically for the studied classification task makes the results more applicable.

After acquisition, the signals were pulse compressed and velocity compensated, the latter in order to compensate for movements across range gates and shift each target into a single virtual gate. The signals were also 
TABLE I: Available measurements in the experimental dataset (No. sequences/ No. segments) distributed over the performed activities.

\begin{tabular}{lllrlll}
\hline & creep & crawl & walk & jog & run & Total \\
\hline toward & $4 / 11$ & $3 / 9$ & $6 / 22$ & $2 / 8$ & $2 / 4$ & $17 / 54$ \\
away & $2 / 5$ & $1 / 2$ & $6 / 23$ & $2 / 7$ & $2 / 4$ & $13 / 41$ \\
\hline Total & $6 / 16$ & $4 / 11$ & $12 / 45$ & $4 / 15$ & $4 / 8$ & $30 / 95$ \\
\hline
\end{tabular}

filtered to remove stationary clutter using an ideal high-pass filter (in frequency domain) with a cut-off velocity of $\pm 0.1 \mathrm{~m} / \mathrm{s}$.

\section{B. Collected Data}

For this paper, data was collected from three test subjects (adult males of average height and build) crawling, creeping on their hands and knees, walking, jogging and running, directly toward or away from the radar at a distance of 20-60 m, on a clear day without rain and on fairly flat and dry ground covered with short grass. In total, 30 data sequences were collected (approximately $12 \mathrm{~s}$ each) and split into time segments of $2.5-3.5 \mathrm{~s}$ each, giving a total of 95 segments, see Table I. Bookkeeping was done to keep record on which time segments that belong to the same sequence.

The available data only cover radial movements, which is a shortcoming. There are important security applications where non-radial motion occurs. However, in many security applications most interesting targets naturally approaches the radar radially or close to radially or the situation can be designed in such a way, e.g., by placing two radars orthogonal to each other. Another argument for using radial data is that the features extracted stem from movements in the targets forward direction; hence, radial data makes a good candidate for evaluating the expressiveness of different features sets, whereas discussions about movements in non-radial angles become less well founded.

In [15] measured data for non-radial movement at $45^{\circ}$ and $90^{\circ}$ were also used. The TVDs of the movements at $45^{\circ}$ are similar to the TVDs at $0^{\circ}$ with some important differences, while the TVDs at $90^{\circ}$ are totally different from the TVDs at $0^{\circ}$ and $45^{\circ}$. These non-radial data could not be used in this article in the same classification experiment due to various reasons and new data are not possible to measure. However, the non-radial data have been used in Section VI-C to discuss and predict the behaviour of the two features/parameters suggested as sufficient in this article, namely the base velocity and the cadence frequency.

\section{Time-Velocity Diagram (TVD)}

The analysis in this article is based on target micro-Doppler signatures, represented as time-velocity diagrams (TVDs). A TVD is the absolute value of a short time Fourier transform (STFT) of the time domain radar signal, see Fig. 1 for examples and [15] for an in principle full set of TVDs. The time domain signal consists of different radar sweeps for the same radar range. The sampling frequency in the time domain is the PRF, thus $17.6 \mathrm{kHz}$.

The STFTs in this article were computed by overlapping FFTs with an integration time of $37.5 \mathrm{~ms}$, a Hamming window and a time step of $5 \mathrm{~ms}$. The integration time is a compromise between the velocity resolution for a constant radial movement (long integration time is good) and the smearing of the TVD caused by radial acceleration (short integration time is good). The chosen integration time is according to the experience of the authors suitable for this kind of target and movement. The chosen time step gives smooth TVD in the time dimension. The windows type is a standard window with low sidelobes. After the STFT the signal magnitude is converted to $\mathrm{dB}$ and this is the TVD used for the feature extraction. 


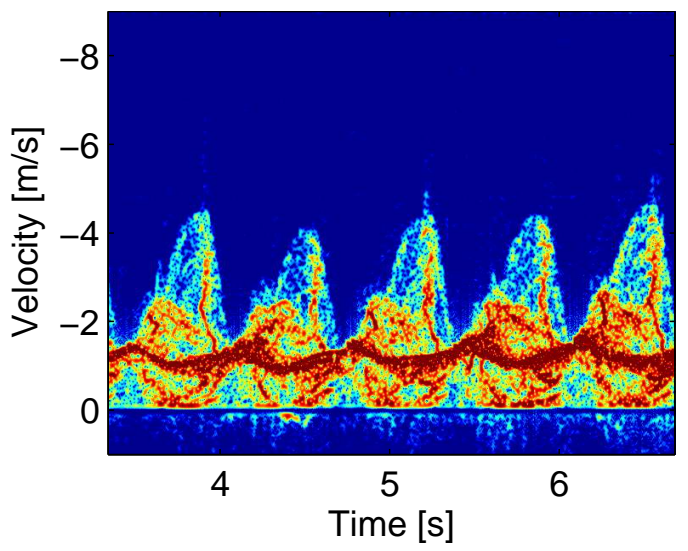

(a) TVD: walking person

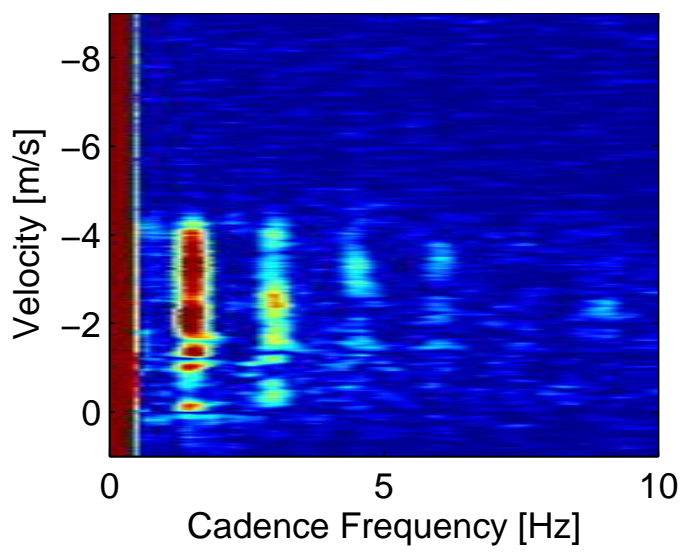

(c) CVD: walking person

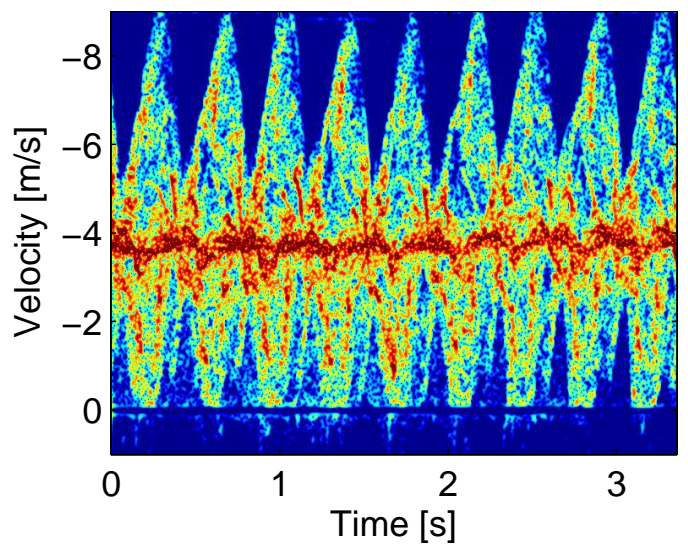

(b) TVD: running person

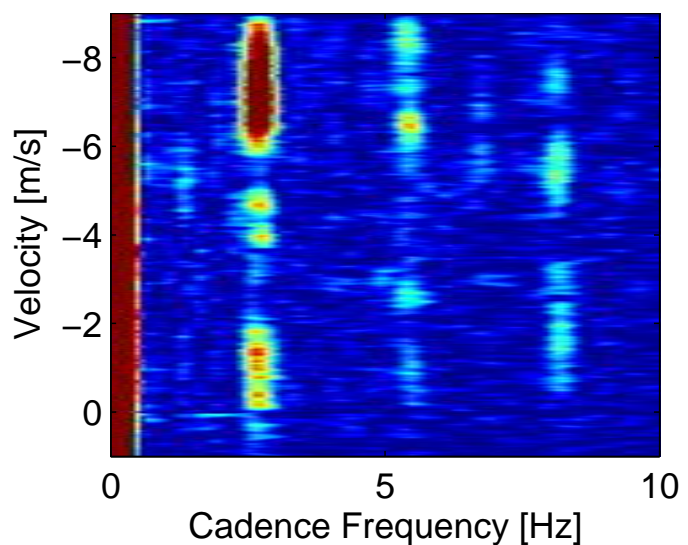

(d) CVD: running person

Fig. 1: Time-velocity diagrams (TVD) and corresponding cadence-velocity diagrams (CVD) for persons walking and running.

\section{Cadence-Velocity Diagram (CVD)}

The article does also make use of cadence-velocity diagrams (CVDs), which are obtained as the absolute value of the Fourier transform of the TVDs with respect to time. Fig. 1 shows TVDs and corresponding CVDs. A CVD shows at which rate different velocities in a TVD repeat ('cadence frequencies'). The CVD characterizes the shape, size, and frequency of the TVD components, which in turn relate to moving parts of the target. CVDs have previously been used to extract features, e.g., $[2,16]$. An interpretation of the CVD is given in $[4,5]$.

\section{E. Ground Truth}

In order to create ground truth information, each data segment has been inspected and manually annotated with the target's base velocity (the velocity of the centre of the mass/main radar cross-section) and main limb cadence/cycle frequency (defined by the cycle time of the micro-Doppler effects produced by the limbs). Fig. 2 depicts the distribution of the acquired values. These two properties will be used in Sec. IV and V to discuss about what are two important information carriers in the dataset. 


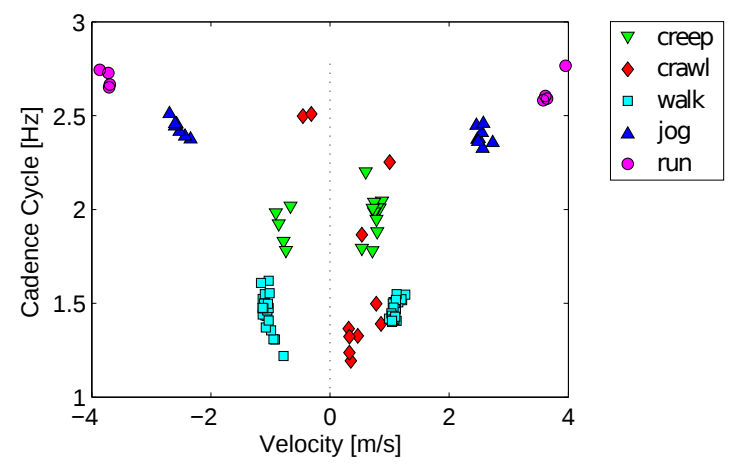

Fig. 2: The distribution of base velocity and limb cadence frequency for all targets within the dataset.

\section{Classification}

Classification is often performed in two steps: feature extraction and feature to class mapping (or just classification).

In the first step, data, $x$, is mapped onto a set of features, $z$, using a feature extractor,

$$
z=F(x)
$$

$x \in \mathbb{R}^{n}$ and $z \in \mathbb{R}^{m}$. In the second step, features, $z$, are mapped onto a discrete set of classes using a classifier,

$$
c=C(z), \quad c \in\left\{\mathcal{C}_{1}, \mathcal{C}_{2}, \ldots, \mathcal{C}_{p}\right\} .
$$

This article applies both these steps, but focus lies on the feature extraction step which is further analysed.

The purpose of the feature extraction is to bring out the discriminating information contained in the data while at the same time suppressing natural variations. This is to simplify the classification task. In the context of this article, features should be robust to movements in the background, moving vegetation, etc., and thereto also be invariant to natural variations between human individuals, to persons carrying objects or not (unless this is of interest), to the direction of movements, and so on.

Two different feature extractors are considered: one by Kim and Ling [1], here denoted the time-velocity (TV) feature method, and one developed at FOI [4, 5], here denoted the cadence-velocity (CV) feature method. The method by Kim and Ling has been chosen for the comparison with the method developed at FOI because they have very similar problem formulations. Both the studied methods are next outlined below.

\section{A. Time-Velocity Features, [1]}

The features considered in this section are extracted from the time-velocity domain, using the method in [1]. The $z^{(\mathrm{TV})}$ comprises six features derived from the TVD representation of the radar signal: $z_{1}$, the torso Doppler frequency; $z_{2}$, the total Doppler bandwidth of the signal; $z_{3}$, the offset of the total Doppler; $z_{4}$, the Doppler bandwidth without micro-Doppler effects; $z_{5}$, the normalized standard deviation of Doppler signal strength; and $z_{6}$, the period of limb motion (i.e., the cadence/cycle frequency). See Fig. 3 and [1] for details.

How to implement these six features is not completely described in [1], hence here follows the interpretations used in this article:

- The upper and lower envelope in the TVD (needed for $z_{2}, z_{3}$, and, $z_{6}$ ), were computed as the top and lower $10 \%$-percentile of the signal energy in each time slot. The noise below a threshold was first removed 


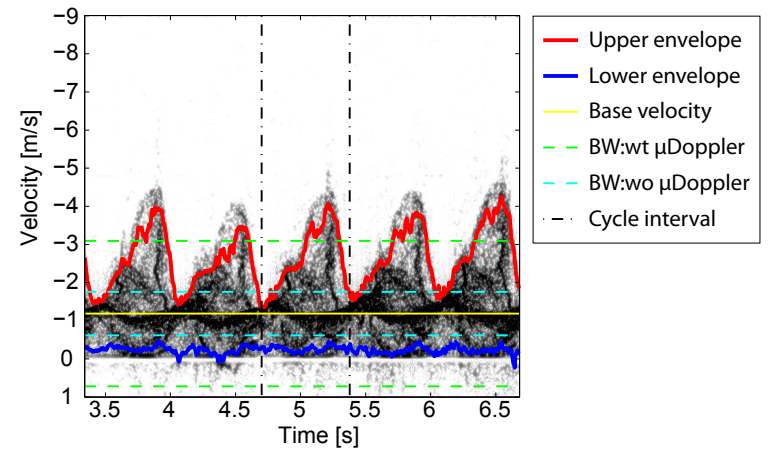

Fig. 3: TV based feature extraction. A TVD with some of the features in the TV method illustrated.

as in [1]. However, the threshold was selected in a different way than in [1] since the method used in [1] did not produce a good enough threshold to be useful in our case.

- The highest and lowest values of the upper and lower envelope $\left(z_{2}\right.$ and $\left.z_{3}\right)$, have been implemented as the $5 \%$ highest/lowest values.

- $z_{5}$ was implemented as the standard deviation of all values in the TVD divided by the average of all the above-noise values in the TVD.

- The period of the limb motion $\left(z_{6}\right)$, was computed by extracting the peaks of the upper envelope and averaging the time between them.

Note that noise thresholding is needed in the TV method to remove the background noise prior to extraction. This tuning parameter is not needed in the CV method described next.

\section{B. Cadence-Velocity Features, [4, 5]}

The CV features, $[4,5]$, described in this section incorporates the strongest parts of the CVD in the feature vector as described below and illustrated in Fig. 4.

1) Compute the total energy, $u\left(f^{c}\right)$, for each cadence frequency, $f^{c}$, in the CVD, i.e., sum up the contribution of all velocities.

2) Choose the $M$ (here $M=3$ ) strongest peaks (cadences frequencies) in $u\left(f^{c}\right),\left\{f_{i}^{c}\right\}_{i=1}^{M}$, and extract the matching velocity profiles (the energy content for the different velocities, $f^{v}$, for the cadence frequency, $\left.f^{c}\right),\left\{v_{i}\left(f^{v}\right)\right\}_{i=1}^{M}$, from the CVD. The choice $M=3$ is taken from [5], where this value gave good results.

3) Compute the base velocity $v_{0}$ as the peak velocity within the total velocity profile (sum the energy for all cadence frequencies).

4) Resample the velocity profiles with linear interpolation, in $L=100$ points, to obtain $\left\{v_{i}\left(f_{i}^{v}\right)\right\}_{i=1}^{L}$. (L is a tuning parameter, for which the value 100 seems to work well.)

5) Normalize the velocity profiles, while maintaining relative magnitudes.

The final feature vector comprises in the given order: the chosen cadence frequencies, the sampled velocity profiles, and finally the base velocity, i.e.,

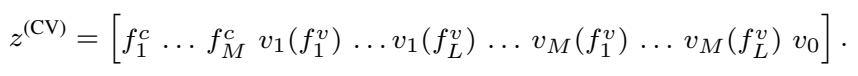




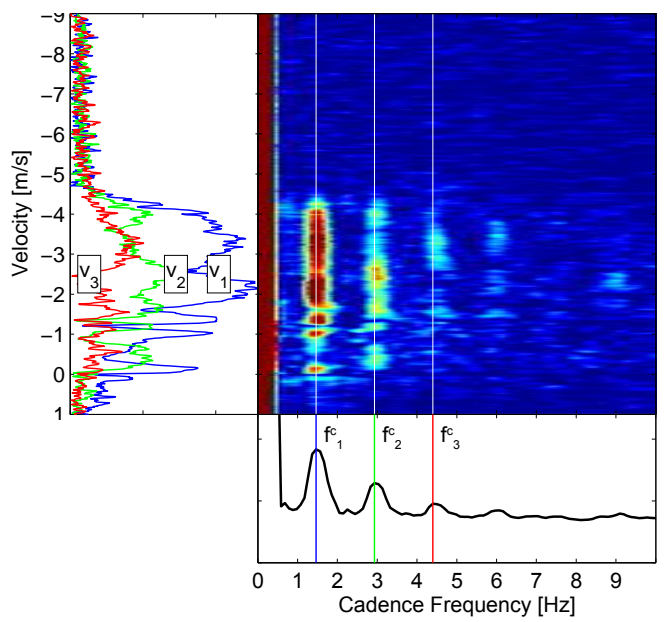

Fig. 4: CV based feature extraction. A CVD with some of the features in the CV method illustrated (cadence frequencies and velocity profiles).

An important difference to the feature vector in [4] is that in [4] the base velocity was not included in the feature vector. But in the current article we want to show the importance of the base velocity for different human activities and therefore it is included here. There were also differences regarding normalization and reversion of the velocity profiles in the feature vector in [4]. Further, in [4] only two cadence frequencies were used. Although the feature selection method in principle was the same as in [4], the feature vector was not the same. It would be an interesting continuation of the presented work to also try to determine automatically how many cadence frequencies are needed.

\section{Classification Algorithm}

In this article support vector machines SVMs [17] are used for the classification task because they have a good reputation and nice properties and because they are frequently used in micro-Doppler classification literature. They have also given good results earlier $[4,5]$ with the CV features used in this article. SVM is a maximum margin classifier, that aims to find hyperplanes that yields the largest possible margin of separation between clusters of data from each respective class. SVM has some nice properties which makes it a suitable choice for this article, namely it can handle linearly non-separable classification problems, multi-class scenarios and multidimensional data. In the future it could be interesting to evaluate different classifiers.

\section{EXPERIMENTAL DETAILS}

In this section the experimental details behind the results presented in this article are given.

\section{A. Feature Extraction}

For each TVD in the dataset, feature vectors were constructed using the two feature extraction methods. The fact that TVDs from the dataset originate from movements in both directions relative to the radar calls for post-processing of the feature vectors. The sign was hence removed from the base velocity, $z_{304}^{(\mathrm{CV})}=\left|v_{0}\right|$, leaving only the speed which avoids that the CV classification method is affected by the direction of movement. The TV features are changed accordingly to make the comparison fair, i.e., the signs are removed from $z_{1}^{(\mathrm{TV})}$ and $z_{3}^{(\mathrm{TV})}$. This slightly improves the performance of the TV method on the considered data. 
TABLE II: Tested SVM kernel functions.

\begin{tabular}{lc}
\hline Kernel & $K(u, v)$ \\
\hline Linear & $u^{T} v$ \\
Polynomial & $\left(\gamma u^{T} v+c_{0}\right)^{d}$ \\
RBF (radial basis function) & $\exp \left(-\gamma|u-v|^{2}\right)$ \\
Sigmoid & $\tanh \left(\gamma u^{T} v+c_{0}\right)$ \\
\hline
\end{tabular}

TABLE III: Optimal SVM parameters as determined by leave one out cross validation and a multi-dimensional grid of candidate parameter settings.

\begin{tabular}{llll}
\hline Features & Kernel & Parameters & Accuracy \\
\hline$F_{\mathrm{CV}}\left(x_{k}\right)$ & Linear & $C=2^{7}$ & $92 \%$ \\
$F_{\mathrm{CV}-}\left(x_{k}\right)$ & RBF & $C=2^{-3}, \gamma=2^{12}$ & $91 \%$ \\
$F_{\mathrm{TV}}\left(x_{k}\right)$ & RBF & $C=2^{5}, \gamma=2^{-3}$ & $88 \%$ \\
\hline
\end{tabular}

Furthermore, to make the feature vectors suitable for the classifier, all features were normalized componentwise to achieve $\left|z_{i}\right| \leq 1$ for all $i$. This is important in order to avoid that features with larger numeric ranges will dominate over features with smaller ranges and to avoid numerical problems [18] .

Finally, and for reasons to be discussed below, a reduced variation of the $\mathrm{CV}$ feature extractor, called the reduced $C V$ method and denoted the $\mathrm{CV}^{-}$method, was designed, which extracts only the $M$ major cadence frequencies (here, $M=3$ ) from the CVD and the base velocity, i.e.,

$$
z^{\left(\mathrm{CV}^{-}\right)}=\left[\begin{array}{lllll}
u_{1} & u_{2} & \ldots & u_{M} & \left|v_{0}\right|
\end{array}\right]
$$

\section{B. Classification: set-up}

The SVM classification was performed using LibSVM [19] and labelled time segments with five activities (disregarding the direction of movement),

$$
c_{i}=\{\text { crawl, creep, walk, jog, run }\}
$$

In addition to the procedure of using a set of samples to train on, the SVM has a few extrinsic parameters that need to be set, namely a cost parameter and a kernel function [20]). For each feature extraction method, a grid-search based strategy was used to find an appropriate kernel (with parameters), and cost-parameter $(C)$. The kernels in Table II were those being evaluated. To make efficient use of the limited set of data, leave-oneout cross validation was applied to evaluate each parameter setting within the grid. The best settings for each method are given in Table III together with the resulting classification accuracy. Accuracy is defined as the correctly classified samples divided by the total number of samples.

\section{Classification: training and validation}

For each set of feature vectors, a classifier was trained using the most appropriate setting as determined above. Due to a limited dataset, resampling was used to train and validate the classifiers. A 'leave-one-sequence-out' cross validation procedure was used, i.e., one sequence at the time was removed from the training dataset and used for validation, and this procedure was repeat for each sequence. This approach was used to minimize the dependency between the training and validation phase. 


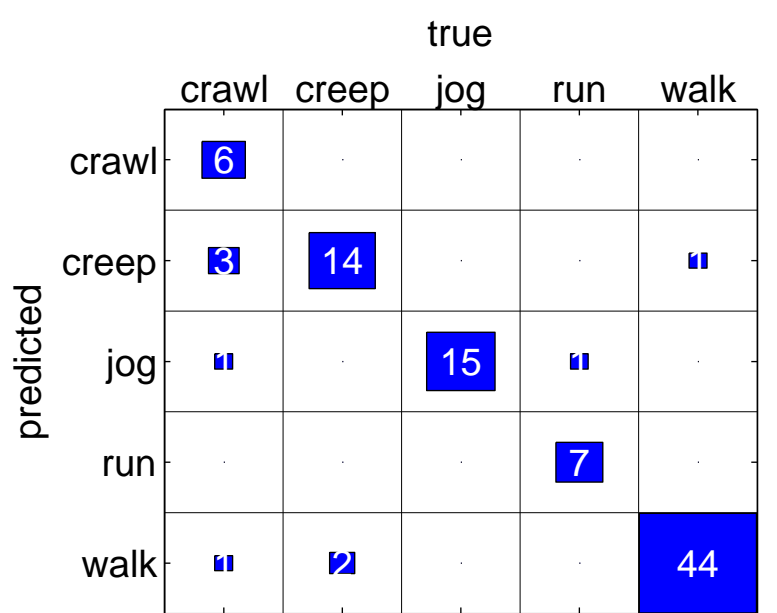

(a) Full CV method, $F_{\mathrm{CV}}\left(x_{k}\right)$. Accuracy: $91 \%$.

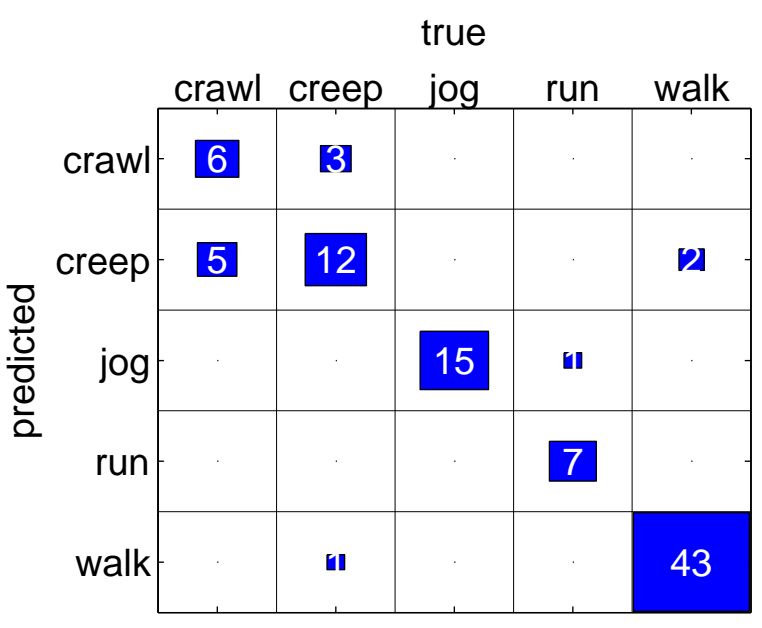

(b) Reduced CV method, $F_{\mathrm{CV}^{-}}\left(x_{k}\right)$. Accuracy: $87 \%$

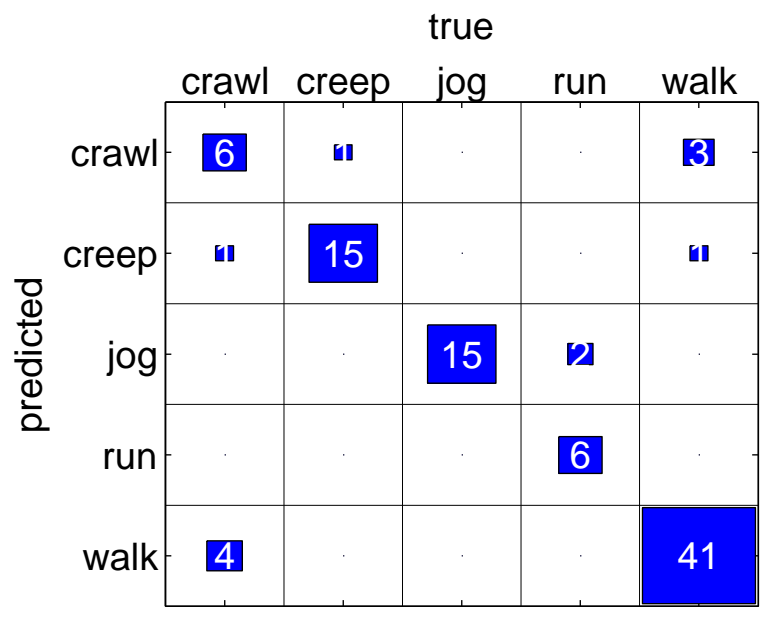

(c) TV method, $F_{\mathrm{TV}}\left(x_{k}\right)$. Accuracy: $87 \%$

Fig. 5: Classification results derived using 'leave-one-sequence-out' validation with 95 instances of data. The numbers in the boxes are the samples classified that way.

\section{Results}

The results from applying the different feature extraction methods and then using an SVM are summarized in Fig. 5. In terms of overall accuracy, the results are comparable to what is reported in e.g., [1]. The dataset indicates that the TV and CV methods have comparable performance and that the base velocity and the major cadence frequencies capture the important differences between the activities. This is indicated by the separation between the classes in Fig. 2 and the performance of the reduced $\mathrm{CV}$ method, $\mathrm{CV}^{-}$, in Fig. 5.

The most difficult activity to classify is crawling; a majority of miss-classified activities involve crawling. The same thing is indicated by Fig. 2, where crawling clusters poorly with instances close to several other activities. This makes it difficult to find a classifier that separates out crawling, at least using the base velocity and cadence frequency. For the $\mathrm{CV}$ based methods $\left(\mathrm{CV}\right.$ and $\left.\mathrm{CV}^{-}\right)$and crawling the most miss-classifications are with creeping. Also in [4] it was difficult to separate crawling and creeping with the CV based method used there. 


\section{DISCUSSION}

In this article we do not try to provide conclusive evidence that the one feature extraction method is better than the other. Instead we provide a qualitative comparison of the two studied methods and what features which seem to provide most information about the human activity. The authors recognize the limited possibility to draw extensive conclusions about feature/classifier performance from the material presented here, partly due to the limited size of the dataset, the activities and the individuals studied. Trustworthy claims about a method's performance require a dataset with many repetitions, individuals, activities, conditions, scenes, directions of motion, etc. However, we think, that for the purpose of the article, the data we have used are sufficient. In this section we will discuss more about sufficient features, weaknesses of the methods and non-radial movement.

\section{A. The base velocity and cadence frequency are sufficient}

It is interesting to discuss the feature extraction methods in light of the dataset being used. The data used in this article represent a quite "normal" scenario with subjects moving with a fix aspect angle, and with "normal" gaits (no excessive arm movements etc.). Under these conditions, the two features (a) the base velocity and (b) the cadence frequency, can provide competitive classification results, see Fig. 2. This is interesting, especially as these properties are implicitly included in both the TV and CV methods. The TV features are mostly related to velocity information, but the period of the limb motion is closely related to the cadence frequency. On the contrary, $\mathrm{CV}$ features focus on cadence frequency information, but the feature vector also include the base velocity.

Thus, the base velocity and cadence frequency are two very descriptive parameters for human gait classification. Therefore, a feature vector containing derivatives of these parameters (such as the studied methods) are likely to perform well in "normal scenarios". A question is then how "rich" a dataset must be in order to thoroughly evaluate feature extractors and classifiers? It is also interesting to determine in which situations a target's base velocity and main cadence frequency are sufficient to classify its activity. It may, in many situations, be advisable to focus on robust estimation of these two parameters instead of developing advanced feature extractors trying to mine-out every detail of a TVD. This aspect has just begun to get attention in the literature. In [21] a method for estimation of base velocity and main cadence frequency based on the cadence frequency and mean velocity of the torso only is suggested. A similar result to ours is found in [6], where the authors note that the cadence frequency and step length ("stride") are two very descriptive parameters for distinguishing between humans and small animals. The paper [22] applies mutual information in order to select features with a good discriminative quality and good feature estimation quality.

\section{B. Problems with the TV and CV methods}

The TV method relies heavily on the upper and lower envelope in the TVD of the target. Our experience is that it can be difficult to automatically estimate these envelopes, especially with low signal-to-noise ratio (SNR). This leaves us to believe that the CV method is more robust as the signal does not have to be separated from the noise (the TV method makes use of a user selected threshold for this, it affects the result, no such user input is needed in the $\mathrm{CV}$ method). The CV method can have a different problem, namely to robustly estimating the cadence frequencies from the CVD, point 2 in Section III-B. These conclusions should be further evaluated and tested with datasets including also non-radial data. Furthermore, the CV method uses a very high-dimensional feature space which, due to the 'curse of dimensionality', is not always wanted. Therefore, the reduced CV 
features set, $\mathrm{CV}^{-}$, comprising only the base velocity and cadence frequencies, was evaluated. With the current dataset, reducing the feature set does not impair the classifier significantly, see Fig. 5.

\section{Non-radial movement}

The cadence frequencies of the CV method and the period limb motion (= the base cadence frequency) of the TV method we believe to be robust up to at least $45^{\circ}$ direction of movement. We believe this by looking at the figures in [15] for a walking and a running person. The envelope of the TVD is clearly seen also for $45^{\circ}$, which would make it as easy to estimate as for $0^{\circ}$ for the TV method and as easy to obtain the correct cadence frequencies for the CV method. See [4, 5] for how the CV method works.

With an non-radial movement the velocity spectrum in Fig. 2 will become more compressed in the velocity dimension. This will make it more difficult to separate the classes. The most affected class separation of this or the only affected is separation of "crawl" from "creep" and "walk" since these classes cannot be separated with the base cadence frequency only. Another complication with non-radial movement is that the trajectory of the torso becomes more unclear in the TVD, see figures for "walk" and "run" in [15]. This will probably lead to a more uncertain estimation of the base velocity and also making it more difficult for the "crawl" class. Thus, there would be more misclassifications involving the "crawl" class for non-radial movement. The classification of the other classes should be fairly robust up to at least $45^{\circ}$.

More extensive experimental data is needed to verify these hypotheses. In order to experimentally verify these we would need to measure on the same moving person simultaneously with several radars in different directions. The TVDs and CVDs of several subsequent measurements, as the ones that we have, vary also because of different human individuals and different movements of the same individual.

An idea for future work would be to replace the base velocity feature with features which are more independent of angle, like the ratio of velocity bandwidth to base velocity, as suggested in [15].

\section{SUMMARY}

In this article two different methods to extract features from micro-Doppler spectrograms, using features in the time-velocity and cadence-velocity domains, have been described and applied to a dataset containing measurements from different human activities. SVM was then used with these features to classify activities, with no significant differences in the classification results. Properties of the extracted features were discussed, and it was shown that comparable classification performance can be obtained using only two properties for the studied dataset, namely the target velocity and the main cadence frequency. This was demonstrated by reducing one of the feature sets. It was noted that these properties are present in both unreduced feature sets, i.e. in both feature selection methods. This suggests that a feature vector with robust estimates of these two properties could be sufficient in many applications. The suggestion must be taken with some caution since the dataset was limited. An interesting direction for future research is to find more robust and efficient ways to construct feature vectors including the target velocity and the main cadence frequency. Also, weaknesses of the methods and the impact of non-radial motion have been discussed.

\section{ACKNOWLEDGEMENTS}

This work has financially been supported by Security Link and the Swedish Foundation for Strategic Research (SSF). We also gratefully thank SAAB AB and especially Mikael Hämäläinen, for cooperation with the measurements. 


\section{REFERENCES}

[1] Kim, Y., Ling, H. "Human activity classification based on micro-Doppler signatures using a support vector machine", IEEE Trans. Geosci. Remote Sens., 47(5), pp. 1328-1337, (May 2009).

[2] Molchanov, P., Astola, J., Egiazarian, K., Totsky, A. "Classification of ground moving radar targets by using joint time-frequency analysis", IEEE Radar Conf., pp. 366-371, (Atlanta, USA2012).

[3] Li, J., Phung, S. L., Tivive, F. H. C., Bouzerdoum, A. "Automatic classification of human motions using Doppler radar", IEEE World Congr. Comp. Intel., (Brisbane, Australia2012).

[4] Björklund, S., Johansson, T., Petersson, H. "Evaluation of a micro-Doppler classification method on mm-wave data", IEEE Radar Conf., (Atlanta, USA2012).

[5] Petersson, H., Björklund, S., Karlsson, M., Lauberts, A. “Towards surveillance using micro-Doppler radar”, Int. Radar Symp., (Hamburg, Germany2009).

[6] Kim, Y., Ha, S., Kwon, J. "Human detection using doppler radar based on physical characteristics of targets", IEEE Geosci. Remote Sens. Lett., 12(2), pp. 289-293, (February 2015).

[7] Bilik, I., Khomchuk, P. "Minimum divergence approaches for robust classification of ground moving targets", IEEE Trans. Aerosp. Electron. Syst., 48(1), pp. 581-603, (January 2012).

[8] Bryan, J., Kwon, J., Lee, N., Kim, Y. "Application of ultra-wide band radar for classification of human activities", IET Radar, Sonar Nav., 6(3), pp. 172 -179, (March 2012).

[9] Fairchild, D., Narayanan, R. "Classification of human motions using empirical mode decomposition of human microdoppler signatures", Radar, Sonar Navigation, IET, 8(5), pp. 425-434, (June 2014).

[10] Bilik, I., Tabrikian, J. "Radar target classification using Doppler signatures of human locomotion models", IEEE Trans. Aerosp. Electron. Syst., 43(4), (October 2007).

[11] Lyonnet, B., Ioana, C., Amin, M. G. "Human gait classification using microDoppler time-frequency signal representations", IEEE Radar Conf., pp. 915-919, (2010).

[12] Tivive, F. H. C., Bouzerdoum, A., Amin, M. G. "A human gait classification method based on radar Doppler spectrograms”, EURASIP J. Adv. in Sig. Proc., 2010, (2010).

[13] Liu, L., Popescu, M., Skubic, M., Rantz, M., Yardibi, T., Cuddihy, P. "Automatic fall detection based on doppler radar motion signature", Pervasive Computing Technologies for Healthcare (PervasiveHealth), 2011 5th International Conference on, pp. 222-225, (2011).

[14] Balleri, A., Chetty, K., Woodbridge, K. "Classification of personnel targets by acoustic micro-doppler signatures", IET Radar, Sonar \& Navigation, 5(9), pp. 943-951, (2011).

[15] Björklund, S., Petersson, H., Nezirovic, A., Guldogan, M. B., Gustafsson, F. "Millimeter-wave radar micro-Doppler signatures of human motion", Int. Radar Symp., (Leipzig, Germany2011).

[16] Ghaleb, A., Vignaud, L., Nicolas, J. M. "Micro-Doppler analysis of wheels and pedestrians in ISAR imaging", IET Sig. Proc., 2(3), pp. 301-311, (2008).

[17] Vapnik, V. The nature of statistical learning theory, 2. edition, (Springer-Verlag Inc, 1999).

[18] Hsu, C.-W., Chang, C.-C., Lin, C.-J. A Practical Guide to Support Vector Classification, Department of Computer Science and Information Engineering National Taiwan University, Taipei 106, Taiwan, (April 15 2010), [Online]. Available: www.csie.ntu.edu.tw/ cjlin/libsvm.

[19] Chang, C.-C., Lin, C.-J. "LIBSVM: A library for support vector machines”, ACM Trans. on Intel. Syst. and Tech., 2 , pp. 27:1-27:27, (2011).

[20] Aizerman, M., Braverman, E., Rozonoer, L. "Theoretical foundations of the potential function method in pattern recognition learning”, Autom. and Remote Contr., 25, pp. 821-837, (1964).

[21] Sun, Z., Wang, J., Yuan, C., Bi, Y., Xiang, H. "Parameter estimation of walking human based on micro-doppler", 12th International Conference on Signal Processing (ICSP2014), pp. 1934 - 1937, (2014).

[22] Gurbuz, S., Tekeli, B., Karabacak, C., Yuksel, M. "Feature selection for classification of human micro-doppler", 
Microwaves, Communications, Antennas and Electronics Systems (COMCAS), 2013 IEEE International Conference on, pp. 1-5, (2013). 